\title{
Bayesian MMSE Estimation of a Gaussian Source in the Presence of Bursty Impulsive Noise
}

\author{
Md Sahabul Alam, Georges Kaddoum, and Basile L. Agba
}

\begin{abstract}
In this letter, we derive the minimum mean square error (MMSE) optimal Bayesian estimation (OBE) for a Gaussian source, in the presence of bursty impulsive noise, as essentially encountered within power substations. Clearly, it is observed that the presence of bursty impulsive noise makes the inputoutput characteristics of MMSE OBE non-linear. To handle the non-linearity, we propose a novel MMSE estimator, based on the detection of the unobservable states of the noise process, using the maximum a posteriori (MAP) detector. Resultantly, the proposed MAP-based MMSE estimator is shown to achieve the lower bound derived for the proposed scenario and outperform the various MMSE estimators that neglect the noise memory.
\end{abstract}

Index Terms-Bursty impulsive noise, Markov process, Bayesian MMSE estimation, BCJR/MAP algorithm.

\section{INTRODUCTION}

The difficulty of estimating a Gaussian source from its available noisy measurements is prevalent in numerous signal processing contexts. In particular, a great deal of prior research is available in the literature, regarding the Gaussian source estimation in the presence of Gaussian noise, in various aspects. In such a scenario [1], the linear MMSE (LMMSE) estimator is proved to be the optimal estimation technique. However, the noise that usually occurs in many environments are highly nonGaussian and display a significant impulsive nature [2]. For example, in power substations, the noise emitted from various power equipment is impulsive [2]-[4]. The impulsive noise measurement campaign in power substation environments also shows that the impulses occur in bursts [3], [4].

On the other hand, the performance of the estimation techniques, in the presence of an impulsive noise, is not widely acknowledged. Banelli considered the MMSE OBE for a Gaussian source impaired by Middleton class-A impulsive noise in [5]. In his work, he showed that the performance of the MMSE OBE strictly depends on the input-output characteristics of the received signal, which becomes non-linear for impulsive noise environments. To tackle this challenge, several MMSE estimators were introduced. The authors in [6] derived the MMSE OBE and its mean-square error (MSE) performance bounds in closed form, assuming that both the noise and the source signals are Gaussian mixture distributed. The obtained results show that the MMSE estimator, under this condition, outperforms the LMMSE estimator. These approaches, however, have a major shortcoming; they ignore

M. S. Alam and G. Kaddoum are with the Electrical Engineering Department, ETS, University of Quebec, Montreal, QC H3C 1K3, Canada (email: md-sahabul.alam.1@ens.etsmtl.ca; geogres.kaddoum@etsmtl.ca).

B. L. Agba is with the Hydro-Qubec Research Institute, Varennes, QC J3X 1S1, Canada (e-mail:,agba.basile@ireq.ca). the inherent memory in the noise process. To address this, Markov chain models have been proposed [7], [8]; in order to better represent the impulsive noise characteristics. Hence, the impact on the performance gain, observed when the memory is exploited in the estimation process, must be evaluated.

To address this issue, the present letter provides a framework for the performance analysis of Bayesian MMSE estimation of a Gaussian source, in the presence of a bursty impulsive noise source. This necessitates a two-step operation: the estimator should be optimal in minimizing the resulting MSE and can detect the state of the noise process simultaneously, thanks to the BCJR algorithm [9] that was found as an effective tool to detect the states of a finite state hidden Markov process. In this letter, we redesign a robust estimator combining these two techniques - MMSE estimation and the BCJR algorithm. As shown in Fig. 1, the MAP detector executes the BCJR algorithm and provides the hard decision of the noise state information to the MMSE estimator. Given the state, the LMMSE estimator is optimal in minimizing the MSE sense.

The contribution of this work is depicted as follows: we derived the MMSE OBE for a scalar Gaussian source estimation in the presence of bursty impulsive noise, modeled by a two-state Markov-Gaussian (TSMG) process [7]. It is shown that similarly to uncorrelated Middleton class-A noise [5], the presence of TSMG noise also makes the input-output characteristics of MMSE OBE non-linear, especially when the environment is more impulsive. To combat the adverse effect of non-linearity, we propose a novel MMSE estimator, based on detecting the unobservable states of the noise process using the MAP state detection. Through the simulation results, the proposed MAP-based MMSE estimator achieves the MSE lower bound derived for the proposed scenario and performs significantly better than the conventional LMMSE estimator, optimized for AWGN environment, and the MMSE estimator that neglects the noise memory.

\section{SYSTEM MODEL}

In this paper, we consider a point-to-point communication system, as shown in Fig. 1. We assume $s_{k}$ is the parameter to be estimated, modeled by a zero-mean Gaussian random variable with variance $\sigma_{s}^{2}$. The received signal at the destination at each time epoch $k$ can be expressed as

$$
y_{k}=s_{k}+n_{k}, \quad k=0,1, \ldots, K-1
$$

where $n_{k}$ is the additive noise, statistically independent of $s_{k}$, and $K$ is the length of the whole received sequence. It is assumed that the noise sample $n_{k}$ follows the TSMG process. The statistical properties of $n_{k}$ are completely defined by the 


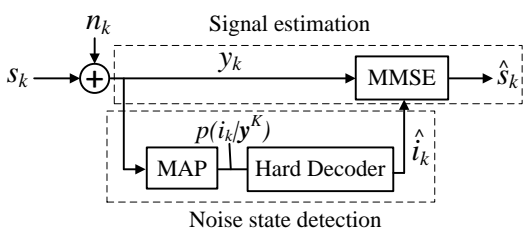

Fig. 1. MAP-based Bayesian MMSE estimation of a Gaussian source in the presence of bursty impulsive noise.

noise state indicator $i_{k} \in\{G, B\}$. In the context of our noise modeling, $G$ and $B$ represent respectively the good and the bad state. The channel is impaired by the Gaussian noise only in the good state, and the bad state occurs when this latter is impaired by impulsive interferers. We model $n_{k}$ as a zeromean Gaussian random variable, so that the probability density function (PDF) of $n_{k}$ conditioned on $i_{k}$ is represented by

$$
f\left(n_{k} \mid i_{k}=m\right)=\frac{1}{\sqrt{2 \pi \sigma_{m}^{2}}} \exp \left(-\frac{n_{k}^{2}}{2 \sigma_{m}^{2}}\right), m \in(G, B),
$$

For this model, the parameter $R=\sigma_{B}^{2} / \sigma_{G}^{2}$ quantifies the impulsive to Gaussian noise power ratio. The statistical description of the state process $\mathbf{i}^{K}=\left\{i_{0}, i_{1}, \ldots, i_{K-1}\right\}$ specifies the channel completely and is evaluated by the state transition probabilities $p_{i_{k} i_{k+1}}=p\left(i_{k+1} \mid i_{k}\right), i_{k}, i_{k+1} \in\{G, B\}$. Using these transition probabilities, the stationary probabilities of being in either the good or the bad state are respectively given by [7],

$$
\pi_{G}=\frac{p_{B G}}{p_{G B}+p_{B G}} \quad \text { and } \quad \pi_{B}=\frac{p_{G B}}{p_{G B}+p_{B G}} .
$$

Also, the parameter $\gamma=\frac{1}{p_{G B}+p_{B G}}$ determines the noise memory and $\gamma>1$ indicates that the channel has a persistent memory.

\section{BAYESIAN MMSE ESTIMATION}

In this section, we consider the MMSE OBE of $s_{k}$, given the observation $y_{k}$. The MMSE OBE corresponds to the posteriori mean [1] and is given by

$$
\begin{aligned}
\hat{s}_{k}\left(y_{k}\right) & =\mathrm{E}\left(s_{k} \mid y_{k}\right)=\mathrm{E}\left[\mathrm{E}\left(s_{k} \mid y_{k}, i_{k}=m\right)\right], \\
& =\sum_{m \in(G, B)} p\left(i_{k}=m \mid y_{k}\right) \hat{s}_{m, k}\left(y_{k}\right),
\end{aligned}
$$

where $\mathrm{E}$ is the expectation operator and $\hat{s}_{m, k}\left(y_{k}\right)=$ $\mathrm{E}\left(s_{k} \mid y_{k}, i_{k}=m\right)$. It should be noted that given $i_{k}=m, s_{k}$ and $y_{k}$ are jointly Gaussian. Hence, LMMSE estimator $\left(\hat{s}_{m, k}\left(y_{k}\right)\right)$ is the optimal estimator of $s_{k}$ and we have [1]

$$
\hat{s}_{m, k}\left(y_{k}\right)=\frac{\sigma_{s}^{2}}{\sigma_{s}^{2}+\sigma_{m}^{2}} y_{k},
$$

where $\sigma_{s}^{2}+\sigma_{m}^{2}$ is the variance of $y_{k}$, given that $i_{k}=m$. Since, $s_{k}$ is independent of $n_{k}$, from the convolution property, the PDF of $y_{k}$ can be represented by

$$
f\left(y_{k}\right)=f\left(s_{k}\right) * f\left(n_{k}\right)=\sum_{m \in(G, B)} \pi_{m} f_{G}\left(y_{k} ; 0, \sigma_{s}^{2}+\sigma_{m}^{2}\right),
$$

where $\pi_{m}=p\left(i_{k}=m\right)$ and $f_{G}\left(y_{k} ; 0, \sigma_{s}^{2}+\sigma_{m}^{2}\right)$ represents a zero-mean Gaussian PDF with variance $\sigma_{s}^{2}+\sigma_{m}^{2}$. Now, from (4), we can deduce that the posteriori probability $p\left(i_{k}=m \mid y_{k}\right)$ is also required to derive the MMSE estimator. Using the Bayes rule, this can be obtained as

$$
p\left(i_{k}=m \mid y_{k}\right)=\frac{\pi_{m} f_{G}\left(y_{k} ; 0, \sigma_{s}^{2}+\sigma_{m}^{2}\right)}{\sum_{j \in(G, B)} \pi_{j} f_{G}\left(y_{k} ; 0, \sigma_{s}^{2}+\sigma_{j}^{2}\right)},
$$

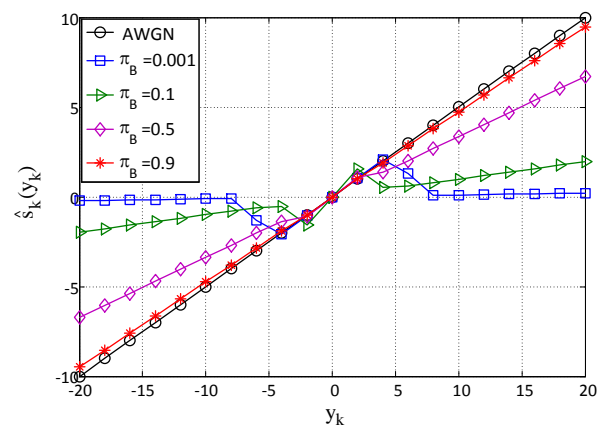

Fig. 2. Impact of the impulsive probability $\pi_{B}$ on the input-output characteristics of MMSE optimal Bayesian estimation. It is assumed that $\sigma_{s}^{2}=1$, $\sigma_{n}^{2}=1, R=100$, and $\gamma=100$.

Hence, substituting equation (5) and (7) into (4), the MMSE OBE of $s_{k}$ given $y_{k}$ is obtained by

$$
\hat{s}_{k}\left(y_{k}\right)=\sum_{m \in(G, B)} \frac{\pi_{m} f_{G}\left(y_{k} ; 0, \sigma_{s}^{2}+\sigma_{m}^{2}\right) \sigma_{s}^{2}}{\sum_{j \in(G, B)} \pi_{j} f_{G}\left(y_{k} ; 0, \sigma_{s}^{2}+\sigma_{j}^{2}\right)\left(\sigma_{s}^{2}+\sigma_{m}^{2}\right)} y_{k} .
$$

Fig. 2 shows the input-output characteristics of MMSE OBE, using equation (8) for different values of the impulsive probability $\pi_{B}$. As observed in Fig. 2, when the value of $\pi_{B}$ increases, the impulsive noise becomes closer to the Gaussian noise and the input-output characteristics of MMSE OBE tend to the well-known LMMSE estimation. On the other hand, when the value of $\pi_{B}$ decreases, the environment becomes more impulsive, as indicated by rare impulsive events, and the input-output characteristic becomes more non-linear. Thus, the presence of bursty impulsive noise introduces non-linearity in the measurement $y_{k}$. This necessitates the designing of a MMSE estimator, in order to achieve a better MSE performance over the conventional LMMSE estimator.

In addition, as well as having to estimate the source signal, if the estimator can detect the states of the impulsive process simultaneously, the conventional LMMSE estimator can be considered as the optimal choice in minimizing the MSE sense [1]. In this vein, in the following section, we will discuss three state detection algorithms. To achieve the best performance, the optimal detector must detect the states of the impulsive noise as accurately as possible.

\section{EXPLOITING STATE INFORMATION}

In this section, we will discuss three state detection algorithms to pursue the explicit use of the noise state information in the MMSE estimation process. Hence, in other words, when the receiver knows whether the impulsive noise is affecting the signal samples or not, the MMSE OBE can be confirmed, according to equation (5). Revisiting equation (5), it can be illustrated by the fact that $\sigma_{m}^{2}$ can be substituted by $\sigma_{B}^{2}$, in case of the presence of an impulsive noise and with $\sigma_{G}^{2}$ otherwise.

\section{A. Genie Detection}

For genie detection, it is assumed that the receiver is deemed to have perfect noise state information. While such an approach is conceptually valuable to provide us a good indication of the best achievable performance, the realization 


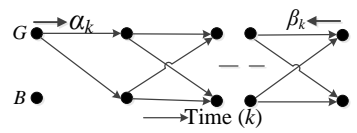

Fig. 3. Trellis representation of the two-state Markov-Gaussian noise model.

of such a detector is very hard, if not impossible. In the context of achievable performance, the following sections provide some algorithms to obtain the states of the noise.

\section{B. MAP-based State Detection using the BCJR Algorithm}

For this scheme, at each $k$, the receiver evaluates the a posteriori probability $p\left(i_{k} \mid \mathbf{y}^{K}\right)$ that the actual channel state is $i_{k}$, given the received sequence $\mathbf{y}^{K}=\left\{y_{0}, y_{1}, \ldots, y_{K-1}\right\}$. This can be obtained as

$$
p\left(i_{k} \mid \mathbf{y}^{K}\right)=\frac{p\left(i_{k}, \mathbf{y}^{K}\right)}{p\left(\mathbf{y}^{K}\right)} \propto p\left(i_{k}, \mathbf{y}^{K}\right),
$$

We now define the following

$$
\begin{aligned}
\alpha_{k}\left(i_{k}\right) & =p\left(y_{0}, y_{1}, \ldots, y_{k-1}, i_{k}\right) \\
\beta_{k}\left(i_{k}\right) & =p\left(y_{k}, y_{k+1}, \ldots, y_{K-1} \mid i_{k}\right) \\
\delta_{k}\left(y_{k}, i_{k}, i_{k+1}\right) & =p\left(i_{k+1}, y_{k} \mid i_{k}\right)=p\left(i_{k+1} \mid i_{k}\right) f\left(y_{k} \mid i_{k}\right),
\end{aligned}
$$

where $\alpha_{k}\left(i_{k}\right)$ and $\beta_{k}\left(i_{k}\right)$ are termed as the forward and backward filters, and $\delta_{k}\left(y_{k}, i_{k}, i_{k+1}\right)$ represents the branch metric of the trellis diagram, as shown in Fig. 3. Using (10) and (11), the probability $p\left(i_{k}, \mathbf{y}^{K}\right)$ in (9) can be written as

$$
\begin{aligned}
p\left(i_{k}, \mathbf{y}^{K}\right) & =p\left(y_{0}, y_{1}, \ldots, y_{k-1}, i_{k}\right) p\left(y_{k}, y_{k+1}, \ldots, y_{K-1} \mid i_{k}\right), \\
& =\alpha_{k}\left(i_{k}\right) \beta_{k}\left(i_{k}\right) .
\end{aligned}
$$

where the first equality comes from the Markov property. Then, the noise state can be expressed as

$$
\hat{i}_{k}= \begin{cases}G & \text { if } L_{i_{k}} \geq 0 \\ B & \text { if } L_{i_{k}}<0\end{cases}
$$

where $L_{i_{k}}$ is the log-likelihood ratio (LLR) and $\hat{i}_{k}$ represents the hard decision of the impulsive noise state at time epoch $k$. For this, the LLR values at the receiver can be computed by

$$
L_{i_{k}}=\ln \left\{\frac{\alpha_{k}\left(i_{k}=G\right) \beta_{k}\left(i_{k}=G\right)}{\alpha_{k}\left(i_{k}=B\right) \beta_{k}\left(i_{k}=B\right)}\right\} \text {. }
$$

Accordingly, the forward and backward filters can be computed recursively as

$$
\begin{aligned}
\alpha_{k+1}\left(i_{k+1}\right) & =\sum_{i_{k}} \alpha_{k}\left(i_{k}\right) \delta_{k}\left(y_{k}, i_{k}, i_{k+1}\right), \\
\beta_{k}\left(i_{k}\right) & =\sum_{i_{k+1}} \beta_{k+1}\left(i_{k+1}\right) \delta_{k}\left(y_{k}, i_{k}, i_{k+1}\right),
\end{aligned}
$$

where the filters recursions are initialized with $\alpha_{0}\left(i_{0}=m\right)=$ $\pi_{m}$, and $\beta_{K}\left(i_{K}=m\right)=1$.

\section{Sample-by-Sample State Detection}

We next consider a state detection algorithm called sampleby-sample state detection scheme. Although aware of the impulsive noise state, it neglects the inherent noise memory. For such a scenario, it is assumed that $\gamma=1$ in the state detection process, which corresponds to the Bernoulli-Gaussian noise [10] instead of TSMG noise. Under this approximation, the above recursive MAP-based noise state detector simplifies to a memoryless detector and the probability of having a state can be computed from the probability, $p\left(i_{k} \mid y_{k}\right)=p\left(i_{k}\right) f\left(y_{k} \mid i_{k}\right)$. Using this, the LLR values are obtained as

$$
L_{i_{k}}=\ln \left\{\frac{\pi_{G} f\left(y_{k} \mid i_{k}=G\right)}{\pi_{B} f\left(y_{k} \mid i_{k}=B\right)}\right\} .
$$

Where $f\left(y_{k} \mid i_{k}=m\right)=f_{G}\left(y_{k} ; 0, \sigma_{s}^{2}+\sigma_{m}^{2}\right)$. From the LLR values, the receiver then determines the noise states according to (14).

\section{AWGN Scenario}

This is the simplest estimation technique since it is blind to the noise states for the estimation process. Under this consideration, the $\mathrm{OBE} \hat{s}_{k}\left(y_{k}\right)$ of $s_{k}$ given $y_{k}$ can be obtained as

$$
\hat{s}_{k}\left(y_{k}\right)=\frac{\sigma_{s}^{2}}{\sigma_{s}^{2}+\sigma_{n}^{2}} y_{k} .
$$

where $\sigma_{n}^{2}=\sum_{m \in(G, B)} \pi_{m} \sigma_{m}^{2}$ denotes the variance of $n$.

\section{E. Complexity Discussion}

It is worth to point out that, despite having a better performance, the complexity of the proposed MAP-based MMSE estimation scheme grows exponentially with the frame length, due to the implementation of the BCJR algorithm, while it grows linearly in case of symbol-by-symbol estimation schemes [11]. However, in Section VI we show that the potential performance gain of this scheme justifies the increase in complexity, which makes this receiver suitable for reliable communication scenarios. On the other hand, if we only consider the forward recursions of the BCJR algorithm, then the complexity problem will probably be reduced with a compromise in its performance. Hence, this scheme exhibits a performance/complexity trade-off.

\section{Performance Analysis}

The performance of this scheme is evaluated in terms of distortion or MSE and it can be obtained by

$$
D \equiv \mathrm{E}\left\{\left(s_{k}-\hat{s}_{k}\right)^{2}\right\}=\int_{s} \int_{y}\left(s_{k}-\hat{s}_{k}\left(y_{k}\right)\right)^{2} f(s, y) d s d y
$$

However, the closed form expression of the integrals in (19) is mathematically intractable which makes it difficult to analytically investigate the MSE. As a result, approximating its bounds remain an alternative solution to evaluate the performance of our proposed scheme. In this vein, a lower bound (LB) is obtained under the hypothetical assumption that there is no uncertainty about the state $i_{k}$, i.e., the genie condition. The LB $\left(D_{L B}\right)$ under this consideration can be obtained as

$$
D_{L B}=\sum_{m \in(G, B)} \pi_{m}\left(\sigma_{s}^{2}-\frac{\sigma_{s}^{4}}{\sigma_{s}^{2}+\sigma_{m}^{2}}\right) .
$$

To derive the upper bound $\left(D_{U B}\right)$, we invoke the LMMSE estimator since this latter obtains the smallest MSE among all the estimators which are linear in the observations [1]. The MSE of the LMMSE estimator for this scheme is

$$
D_{U B}=\sigma_{s}^{2}-\frac{\sigma_{s}^{4}}{\sigma_{s}^{2}+\sigma_{n}^{2}} \text {. }
$$




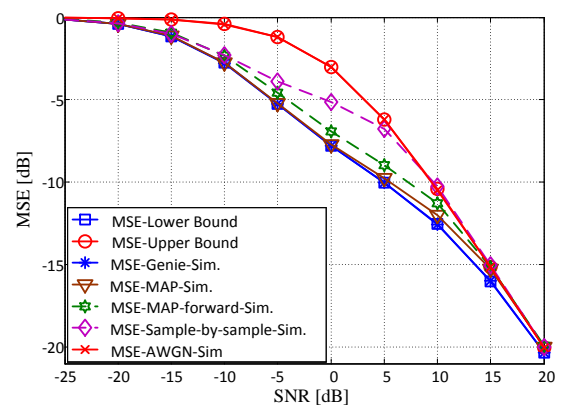

Fig. 4. Analytical and simulated MSE performances of different estimation techniques against the SNR. It is assumed that $\pi_{B}=0.1, R=100$, and $\gamma=100$.

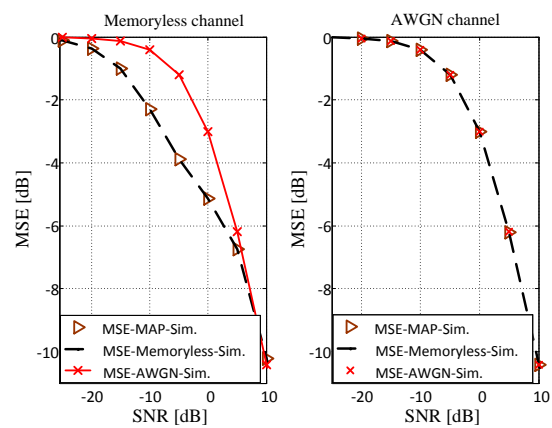

Fig. 5. MSE performances of different estimation techniques against the SNR. It is assumed that $\pi_{B}=0.1$ with $\gamma=1, R=100$ for the memoryless channel, and $\gamma=1, R=1$ in case of AWGN channel.

\section{Numerical RESULtS}

In this section, we simulate the MSE performances of the proposed scenario to confirm the analytical results. It is required to estimate the source parameter $s_{k}$, which is modeled as a Gaussian random variable, with variance $\sigma_{s}^{2}=1$. Also, the impulsive noise that corrupts the source signal is characterized by the parameters $\pi_{B}=0.1, \gamma=100$, and $R=100$ [7]. The MSE performances are calculated against average SNR. Here, the average $\mathrm{SNR}$ is defined as $\mathrm{SNR}=\sigma_{s}^{2} / \sigma_{n}^{2}$.

Fig. 4 shows the simulated MSE performances of different estimation techniques. The simulated MSE performances are obtained by calculating the sample means of $\left(s_{k}-\hat{s}_{k}\left(y_{k}\right)\right)^{2}$ using $2 \times 10^{5}$ frames with $K=10^{3}$ samples of each frame. In Fig. 4, the simulation result attained under genie aided estimation perfectly matches the lower bound is well displayed. However, the genie detection is practically infeasible. Interestingly, from the figure, it is also observed that the proposed MAP-based MMSE estimator almost approaches the performance of genie aided scheme and performs significantly better than the conventional schemes. It obtains a minimum SNR gain of around $8 \mathrm{~dB}$ over the LMMSE estimator and around $5 \mathrm{~dB}$ over the sample-by-sample estimator, at the expense of a higher complexity, due to the BCJR algorithm. Moreover, by considering the forward recursions of the BCJR algorithm, a tight performance gap with the original scheme is observed, while the complexity problem is reduced. This confirms that significant performance gains can be obtained when the noise memory is utilized in the estimation process. In addition, simpler sample-by-sample-based estimator still exhibits better performance than the LMMSE scheme, by uti- lizing the impulsive noise statistics in the estimation process. Finally, the LMMSE leads to the worst performances.

Fig. 5 also shows the simulated MSE performances of different estimation techniques. The essence of plotting Fig. 5 is to visualize how the proposed MAP-based MMSE estimator behaves over the memoryless and AWGN channel. From Fig. 5, it can be inferred that both the MAP-based and sample-bysample-based estimations show the same performance, when we consider $\gamma=1$ in the noise process. These results confirm that the optimal MAP detector simplifies to the memoryless detector when the considered impulsive noise is memoryless. Again, the LMMSE estimator attains around $5 \mathrm{~dB}$ worse performance over them in these impulsive environments, in low SNR region. Finally, the three estimators obviously exhibit the same performance over AWGN channel.

\section{CONCLUSION}

In this letter, we have provided the necessary theoretical foundation for optimal Bayesian estimation of a scalar Gaussian source, in the presence of bursty impulsive noise. It is shown that the presence of bursty impulsive noise makes the input-output characteristics of MMSE OBE non-linear. To combat the effect of non-linearity, we have proposed a novel estimation technique based on detecting the unobservable states of the noise process. The simulation results confirmed that the proposed MAP-based Bayesian MMSE estimator outperforms the various MMSE estimators employing memoryless estimation. Noteworthy, the proposed estimator can be easily adapted to any Gaussian source estimation in the presence of any Gaussian mixture noise with memory. Future extension of this work may include deriving the exact closed form expression for the MSE.

\section{REFERENCES}

[1] S. M. Kay, Fundamentals of statistical signal processing, volume I: estimation theory. Englewood Cliffs, NJ: Prentice Hall, 1993.

[2] D. Middleton, "Statistical-physical models of electromagnetic interference," IEEE Trans. Electromagn. Compat., vol. EMC-19, no. 3, pp. 106127, 1977.

[3] I. Portuguds, P. J. Moore, and I. Glover, "Characterisation of radio frequency interference from high voltage electricity supply equipment," in Proc. 12th Int. Conf. Antennas Propag., 2003, vol. 2, pp. 820-823.

[4] F. Sacuto, B. L. Agba, F. Gagnon, and F. Labeau, "Evolution of the RF characteristics of the impulsive noise in high voltage environment," in Proc. IEEE 3rd Int. Conf. Smart Grid Commun., 2012, pp. 686-691.

[5] P. Banelli, "Bayesian estimation of a Gaussian source in Middleton's class-A impulsive noise," IEEE Signal Process. Lett., vol. 20, no. 10, pp. 956-959, 2013.

[6] J. T. Flam, S. Chatterjee, K. Kansanen, and T. Ekman, "On MMSE estimation: A linear model under Gaussian mixture statistics," IEEE Trans. Signal Process., vol. 60, no. 7, pp. 3840-3845, 2012.

[7] D. Fertonani and G. Colavolpe, "On reliable communications over channels impaired by bursty impulse noise," IEEE Trans. Commun., vol. 57, no. 7, pp. 2024-2030, 2009.

[8] M. S. Alam, F. Labeau, and G. Kaddoum, "Performance analysis of DF cooperative relaying over bursty impulsive noise channel," IEEE Trans. Commun., vol. 64, no. 7, pp. 2848-2859, 2016.

[9] L. Bahl, J. Cocke, F. Jelinek, and J. Raviv, "Optimal decoding of linear codes for minimizing symbol error rate," IEEE Trans. Inf. Theory, vol. 20, no. 2, pp. 284-287, 1974.

[10] M. Ghosh, "Analysis of the effect of impulse noise on multicarrier and single carrier QAM systems," IEEE Trans. Commun., vol. 44, no. 2, pp. 145-147, 1996.

[11] D. Fertonani, A. Barbieri, and G. Colavolpe, "Reduced-complexity BCJR algorithm for turbo equalization," IEEE Trans. Commun., vol. 55, no. 12, pp. 2279-2287, 2007. 